\title{
Network Pharmacology and Experiment Verification to Explore the Potential Mechanism of Yin-Huo-Tang for Lung Adenocarcinoma Recurrence
}

Dianna Liu

Beijing University of Chinese Medicine https://orcid.org/0000-0002-0085-8854

Shicheng Lin

Beijing University of Chinese Medicine

Yuan Li

Beijing University of Chinese Medicine

Tian Zhou

Dongfang Hospital, Beijing university of Chinesse medicine

Kaiwen Hu ( $\nabla$ kaiwenh@163.com )

Beijing University of Chinese Medicine

\section{Quanwang Li}

Dongfang hospital,Beijing University of Chinese Medicine

\section{Research}

Keywords: Yin-Huo-Tang, Lung adenocarcinoma recurrence, network pharmacology, Sphingolipid signaling pathway, Traditional Chinese medicine

Posted Date: October 12th, 2021

DOl: https://doi.org/10.21203/rs.3.rs-956330/v1

License: (c) (i) This work is licensed under a Creative Commons Attribution 4.0 International License. Read Full License 


\section{Abstract}

\section{Background}

Lung adenocarcinoma (LUAD) is one of the most common malignancies with a rise in new cases worldwide each year. Recurrence significantly influences the survival in patients with LUAD. Yin-Huo-Tang (YHT) is a classic traditional Chinese prescription, used to prevent lung cancer relapse by "nourishing yin and clearing heat".

\section{Methods}

In this study, the mechanism of YHT in LUAD recurrence was investigated. Firstly, the bioactive compounds-targets network and the protein-protein interaction network were constructed, and functional annotation and pathway enrichment analyses were performed. Pivotal compounds and hub genes were selected from the networks. Subsequently, the effectiveness of YHT was confirmed in lewis lung carcinoma mice. RNA sequencing was used to explore the mRNA expression differences between tumor tissues in the model mouses and YHT-treated mouses. The pathways screened by network pharmacology and RNA sequencing analysis at the same time were considered the most important pathways. At last, qualitative phytochemical analysis, molecular docking technology, PCR and WB analysis were used to validate the pivotal active ingredients, hub genes and main pathways.

Results

There were 128 active compounds, 419 targets interacting with LUAD recurrence. Network analysis identified 4 pivotal compounds, 28 hub genes and 30 main pathways. Target genes mainly focused on inflammation, metabolism, immune responses and apoptosis. We confirmed that YHT could inhibit the recurrence of lung adenocarcinoma through animal experimental study. Sphingolipid signaling pathway was the common main pathway in network pharmacology and RNA sequencing results. The hub genes related with the sphingolipid signaling pathway was S1PR5. Qualitative phytochemical analysis of the water extract of YHT confirmed the presence of 3 pivotal compounds, namely stigmasterol, nootkatone and ergotamine. The results of molecular docking verified the pivotal compounds of YHT could good affinity with the S1PR5. The PCR and WB analysis verified YHT suppressed lewis lung cancer cells proliferation by inhibiting S1P/S1PR5/Gi/Ras/Raf/MEK/ERK pathway, and inhibited migration through S1P/S1PR5/Gi/PI3K/RAC pathway.

\section{Conclusion}

The results confirmed the therapeutic effect of YHT on the recurrence of LUAD by multi-component-multitarget mode, the sphingolipid signaling pathway was one of the most relevant potential signaling pathways.

\section{Background}


Lung adenocarcinoma (LUAD) is one of the most common malignancies with a rise in new cases worldwide each year. Early diagnosis and surgical treatment are of great importance [1]. Undergoing surgery for early-stage patients and performing adjuvant treatments according to the conditions of the patient are recommended, but about $30-50 \%$ of early-stage lung cancer patients would die within 5 years of recurrent diseases [2]. The pathogenesis of LUAD recurrence is closely related to lipid metabolism alterations [3], immune infiltration [4, 5], gene mutations [6, 7], cell proliferation [5], enhanced stemness, DNA repair deficiency, bacterial microbiome [8] and angiogenesis $[9,10]$. In the theory of traditional Chinese medicine (TCM), LUAD belongs to the category of "lung obstruction", which can be treated by "nourishing yin and clearing heat". Various Chinese herbal medicines and decoctions, such as Rabdosia rosthornii (Diels) H.Hara [11], Tripterygium Hook.f. [12], Ze-Qi-Tang [13] and Fei-Liu-Ping ointment [14] have been reported to inhibit autophagy and apoptosis of tumor cell, angiogenesis and epithelial to mesenchymal transition (EMT) outside the tumor $[15,16]$. Thus, TCM has advantage in suppressing recurrence of lung cancer postoperative patients $[17,18]$.

Yin-Huo-Tang (YHT) is famous for clearing "heat" and nourish "yin" in TCM, which is first published in Chen shiduo's "Bian Zheng Qi Wen" of the Chinese Qing Dynasty. It consists of the Rehmannia glutinosa (Gaertn.) DC. (Chinese name:Shudi), the Morinda officinalis F.C.How (Chinese name:Bajitian), the Poria cocos (Schw.) Wolf (Chinese name: Fuling), the Ophiopogon japonicus (Thunb.) Ker Gawl. (Chinese name:Maidong) and the Schisandra chinensis (Turcz.) Baill. (Chinese name:Wuweizi). Currently, YHT is used to treat lung cancer [19-21] based on the function of "nourishing yin and clearing heat". However, the mechanism of YHT for treatment of LUAD remains unclear, impeding progress in clinical usage of YHT. Further studies are therefore necessary to explore the mechanisms involved.

Network pharmacology is an emerging strategy based on multi-disciplinary technologies. In this approach, the multiingredient-multitargets mechanism of herbs can be revealed systematically $[22,23]$.

In the study, the bioactive compounds-targets network and the protein-protein interaction (PPI) network were firstly constructed, then Gene Ontology (GO) and Kyoto Encyclopedia of Genes and Genomes (KEGG) enrichment were performed[24], in order to find the pivotal compounds, hub genes, functional annotation and main pathways, which elucidated the molecular mechanism of YHT during LUAD recurrence. Subsequently, lewis lung carcinoma mice were used to confirm the effectiveness of YHT. And the differential expression genes (DEGs) induced by YHT therapy was analyzed by RNA sequencing in lewis lung cancer mouse model. KEGG enrichment also played on DEGs in order to find the common main pathways. At last, we proved the presence of pivotal compounds by qualitative phytochemical analysis, confirmed the hub genes which related with the main pathway by molecular docking, and verified the main pathway through PCR and WB analysis (Figure 1).

\section{Methods}

Methods 


\section{Candidate Compounds and Targets Screening}

All of the chemical ingredients and targets of YHT were collected from the Traditional Chinese Medicine Systems Pharmacology database and Analysis Platform (TCMSP, http://tcmspw.com, updated in May 2020), Bioinformatics Analysis Tool for Molecular Mechanism of Traditional Chinese Medicine (BATMANTCM, http://bionet.ncpsb.org.cn/batman-tcm/, updated in May 2020) and wide-scale searches of the literature [24]. Here, we selected as candidate compounds and targets that met the criteria of oral bioavailability (30\%) and drug similarity (0.14) in TCMSP, and score cutoff $>30$, adjusted $p<0.05$ in BATMAN-TCM for further analysis.

\section{Identification of Disease Targets}

We retrieved LUAD recurrent protein targets from GeneCards (https://www.genecards.org/, updated in May 2020) and OMIM database (https://omim.org/, updated in May 2020) [24]. Overlapping targets from drug and disease were obtained for further study. All the targets were standardized through UniProt [25]. Functional annotation and pathway enrichment analyses

The Database for Annotation, Visualization, and Integrated Discovery (DAVID) v.6.8 (https://david.ncifc rf.gov) was used for GO and KEGG enrichment analyses of the detected targets[24]. P value $<0.05$ was set as statistically significant.

\section{PPI Data}

We used the Search Tool for the Retrieval of Interacting Genes (STRING, www.string-db.org/) database to construct a PPI network of the detected targets. And interaction score $>0.9$ was considered statistically significant. Furthermore, the result of the PPI network was imported into the Cytoscape (verson 3.7.1) plugin to create network visualizations and screened the hub genes with cytoHubba plugin[26, 27].

\section{Network Construction}

Cytoscape (verson 3.7.1) software was used to construct drug-active ingredients- hub genes-disease network diagrams.

\section{Preparation and Qualitative Phytochemical Analysis of YHT}

YHT granule was purchased from Beijing Tcmages Pharmaceutical Co., LTD (Beijing, China). It consists of five common Chinese herbal ectracts: Rehmannia glutinosa (Gaertn.) DC., the Morinda officinalis F.C.How, the Poria cocos (Schw.) Wolf, the Ophiopogon japonicus (Thunb.) Ker Gawl. and the Schisandra chinensis (Turcz.) Baill., as listed in Table1. The five granules were mixed in water with a ratio of 30:10:10:5:2. Chromatographic separation was accomplished in an Thermo Ultimate 3000 system. We used the methods described in the Chinese Pharmacopoeia. 
Table 1

Compositions of YHT

\begin{tabular}{|c|c|c|c|c|c|}
\hline Latin name & $\begin{array}{l}\text { Chinese } \\
\text { name }\end{array}$ & $\begin{array}{l}\text { Dose of } \\
\text { herbal } \\
\text { medicine(g) }\end{array}$ & $\begin{array}{l}\text { Dose of } \\
\text { granules(g) }\end{array}$ & $\begin{array}{l}\text { Leaching } \\
\text { rate of } \\
\text { granules } \\
\text { in water }\end{array}$ & Production and lot number \\
\hline $\begin{array}{l}\text { Rehmannia } \\
\text { glutinosa } \\
\text { (Gaertn.) DC. }\end{array}$ & Shudi & 90 & 9 & $23.3 \%$ & Henan(China) 19007601 \\
\hline $\begin{array}{l}\text { Morinda } \\
\text { officinalis } \\
\text { F.C.How }\end{array}$ & Bajitian & 30 & 3 & $10 \%$ & Guangdong(China)18031421 \\
\hline $\begin{array}{l}\text { Poria cocos } \\
\text { (Schw.) Wolf }\end{array}$ & Fuling & 30 & 3 & $10 \%$ & Fuling(China)19014801 \\
\hline $\begin{array}{l}\text { Ophiopogon } \\
\text { japonicus } \\
\text { (Thunb.) Ker } \\
\text { Gawl. }\end{array}$ & Maidong & 15 & 1.5 & $26 \%$ & Sichuan(China) 19010501 \\
\hline $\begin{array}{l}\text { Schisandra } \\
\text { chinensis } \\
\text { (Turcz.) } \\
\text { Baill. }\end{array}$ & Wuweizi & 6 & 0.6 & $N A^{*}$ & Liaoning(China)19013262 \\
\hline
\end{tabular}

\section{Chemical Reagents}

Cisplatin was obtained from QILU PHARMACEUTICAL (Shandong, China).

\section{Cell culture}

Lewis lung cancer cells were obtained from the Cell Bank of Chinese Academy of Sciences (Shanghai, China).

\section{Animals and Experimental Groups}

Fifty SPF C57BL/6 mice (50 males) weighing between $18 \mathrm{~g}$ and $22 \mathrm{~g}$ were purchased from the SPF (Beijing) Biotechnology Co.,Ltd. (Beijing China, License No. SYXK 2019-0010). The animal experiment was approved by the Animal Ethics Committee of the SPF (Beijing) Biotechnology Co.,Ltd. on November 15, 2020 (No. AW2020111501), as showed in Additional file 1: Figure S1.

The animals were randomly divided into five groups ( $n=10 /$ group): model (Mod), Cisplatin (Cis), YHT low-dose (YHT-L), YHT medium-dose (YHT-M) and YHT high-dose (YHT-H). The high dose is the dose documented in "Bian Zheng Qi Wen". For the dose recorded in the book was already higher than the doses of other Chinese herbal medicine prescriptions, the ratio of the three doses of high, medium and low was 4:2:1. 
Lewis lung cancer cells $\left(2 \times 10^{6}\right.$ in $200 \mu \mathrm{L}$ serum-free DMEM) were injected s.c. into the right armpit of mice. The subcutaneous tumors were removed 14 days after lewis lung cancer cells injection. Then, all the mouses were given the drug treatment for 14 days. The Mod group and Cis group received normal saline (10 ml/kg/d, intragastric). The Cis group was given cisplatin $(3 \mathrm{mg} / \mathrm{kg} / \mathrm{d}$, intraperitoneal injection) once a week, all the other groups were given the same volume of saline (intraperitoneal injection). Group YHT-L, YHT-M and YHT-H received YHT $(5.5575 \mathrm{~g} / \mathrm{kg} / \mathrm{d}, 11.115 \mathrm{~g} / \mathrm{kg} / \mathrm{d}$, and $22.23 \mathrm{~g} / \mathrm{kg} / \mathrm{d}$, respectively, intragastric, calculated by dose of herbal medicines). On day 28 after the injection of tumor cells, all animals were sacrificed to collect relevant samples.

\section{Immunochemistry}

Tumor tissues were completely stripped after all the animals had been sacrificed. One side of the tumor tissues was used for immunochemistry staining, according to methods described in previous study[28]. Primary antibody used was anti-Ki-67 (abcam, USA). The other tumor tissues were prepared for further analysis.

\section{RNA Sequencing}

RNA Sequencing was based on the Illumina Novaseq 6000 platform. The sequencing experiment used the Illumina TruseqTM RNA sample prep Kit method for library construction. DEGs between the tumor samples of Mod group and YHT-H group were screened by EdgeR. DAVID v.6.8 was used to perform KEGG pathway enrichment analysis.

\section{Real-Time Quantitative Polymerase Chain Reaction (PCR)}

Three tumor tissues were randomly selected from each group for real-time quantitative PCR, according to the methods described in our previous study [29]. The primer sequences of relative genes were listed in Talbe 2. 
Table 2

The primer sequences of relative genes.

\begin{tabular}{|lll|}
\hline Gene & Forward & Reverse \\
\hline B-actin & 5'-CTACCTCATGAAGATCCTGACC-3' & 5'-CACAGCTTCTCTTTGATGTCAC-3' \\
\hline S1P & 5'-GACTTCATGGATCATCCGTTTG-3' & 5'-AAAAGCGAGCGATGTTATCTTC-3' \\
\hline S1PR2 & 5'-TCTCTATGCTAAGCACTACGTG-3' & 5'-GATGAAAACACCCAGTACGATG-3' \\
\hline S1PR5 & 5'-TCTTGCTATTACTGGATGTCGC-3' & 5'-GTGAAGGTGTAGATGATGGGAT-3' \\
\hline Gi & 5'-CTAAAGAGGCGAGGAAAGTCAG -3' & 5'-GGATCCTCATCTGTTTGACGAT -3' \\
\hline Ras & 5'-TGCCTTCTAGAACAGTAGACAC-3' & 5'-CTTTGCTGAGGTCTCAATGAAC-3' \\
\hline Raf & 5'-ACATCAACAACCGAGACCAGATCATC-3' & 5'-CACAGTCAGCCACCAACCTCTTC-3' \\
\hline MER & 5'-GACTTTGAGAAGATCAGCGAAC-3' & 5'-GTTTGATCTCCAGGTGGATCAG-3' \\
\hline ERK & 5'-ATCTCAACAAAGTTCGAGTTGC-3' & 5'-ATGGTGTGCTCTGCTTATGATA-3' \\
\hline PI3K & 5'-AAACAAAGCGGAGAACCTATTG-3' & 5'-TAATGACGCAATGCTTGACTTC-3' \\
\hline RAC & 5'-CCACTGTCCCAATACTCCTATC-3' & 5'-CTTCTTCTCCTTCAGCTTCTCA-3' \\
\hline
\end{tabular}

\section{Western Blot}

Three tumor tissues were randomly selected from each group for western blot analysis, according to the methods described in our previous study [29]. Primary antibodies used were: S1P (Bioss, Beijing, China); S1PR2 (Proteintech, Wuhan, China); S1PR5 (Proteintech, Wuhan, China); GNAl1 (Proteintech, Wuhan, China); Rac1 (Proteintech, Wuhan, China); KRAS1 (Proteintech, Wuhan, China); ERK1 (Proteintech, Wuhan, China); RAF1 (Proteintech, Wuhan, China); Phospoho-ERK1 (Proteintech, Wuhan, China); MEK-1 (Santa, USA); Phospho-Raf1 (abcam, USA); Phospho-MEK1 (abcam USA); actin (Proteintech, Wuhan, China).

\section{Molecular Docking}

The two-dimensional structures of the pivotal compounds were obtained from PubChem (https://pubchem.ncbi.nlm.nih.gov/). ChemBio3D (version 19.0.0.22) software was used to get threedimensional structures of the pivotal compounds, and the structure was optimized according to minimize energy (minimum RMS gradient was 0.01). The crystal structure of the protein was obtained from RCSB PDB (https://www.rcsb.org/). The solvent and organic of the protein were removed by PyMOL. AutoDock

Tools (version 1.5.6) was used to isolate protein and to get active grid box. The compound and protein were docked by Vina, and presented by PyMOL. The binding energy $<-5.0 \mathrm{kal} / \mathrm{mol}$ was considered to have a stable binding conformation.

\section{Statistical Analysis}

The calculated results were mean \pm standard deviation of at least 3 independent experiments. All the statistical analysis was done using SPSS20.0 (SPSS Inc., Chicago, IL, USA). P $<0.05$ was considered 
statistically significant. The differences between the groups were examined using ANOVA, chi-square test and two-tailed unpaired Student's t-test.

\section{Results}

\section{Compound Information}

A total of 149 candidate compounds were selected through TCMSP and BATMAN-TCM databases, of which 23 only from Bajitian, 12 only from Fuling, 16 only from Maidong, 78 only from WuWeizi, 1 common from Bajitian and Shudi, 17 common from Fuling and Wuweizi, 2 common from Shudi and Maidong, as listed in Additional file 2: Table S1.

\section{Targets in YHT Active Against LUAD recurrence}

A total of 111,170 targets directly and indirectly related to LUAD relapse were obtained and $487 \mathrm{YHT}$ related objectives were obtained (Additional file 3: Table S2). A total of 419 shared targeted were obtained (Figure 2A, Additional file 3: Table S2).

There were 128 active ingredients correlated with the 419 common targets. Active ingredients-gene symbols network was shown in Additional file 4: Figure S2 (Additional file 5: Table S3). And four pivotal active ingredients were identified with degree $\geq 46$, namely stigmasterol, epiguaipyridine, nootkatone, ergotamine.

\section{Construction of PPI network and identification of hub genes}

The PPI relationship of 419 target genes was obtained with STRING tool, and the minimum interactive score was 0. 9 (Figure 2B). The genes evaluated by maximal clique centrality ( $>87178291119$, Figure 2C) and the degree ( $>22$, Figure 2D) were identified: S1PR5, PTGER3, OPRM1, OPRK1, MTNR1B, MTNR1A, HTR1D, HTR1B, HTR1A, DRD4, DRD3, DRD2, CXCR4, CX3CR1, CNR2, CNR1, CHRM2, ANXA1, ADRA2C, ADRA2B, ADRA2A, ADORA3, ADORA1, ADCY1, ADRB2, F2, PIK3CA, PIK3R1.

\section{Drug-Active ingredients-Hub Genes-Disease Network Analysis}

Drug-active ingredients- hub genes-disease network was shown in Figure 3, and the more details were listed in Additional file 6: Table S4. There were 5 Chinese herbs, 48 active ingredients and 28 hub genes in the network.

\section{GO and KEGG Pathway Enrichment Analyses}

The 419 common target genes were enriched with GO and KEGG analysis. It turned out that the main biological processes were "oxidation-reduction process", "response to hypoxia", "regulation of postsynaptic membrane potential", "regulation of ion transmembrane transport", "G-protein coupled receptor signaling pathway" and "coupled to cyclic nucleotide second messenger"; the cellular 
component terms were enriched in "plasma membrane", "integral component of plasma membrane", "voltage-gated sodium channel complex", "GABA-A receptor complex and postsynaptic membrane"; the molecular function terms were enriched in "drug binding", "extracellular ligand-gated ion channel activity", "L-ascorbic acid binding", "GABA-A receptor activity" and "voltage-gated sodium channel activity". In terms of signaling pathway enrichment analysis, we found target genes mainly focused on inflammation, metabolism, immune responses, and apoptosis, such as "Purine metabolism (hsa00230)", "AMPK signaling pathway (hsa04152)", "Human cytomegalovirus infection (hsa05163)", "Apoptosis (hsa04210)", "Insulin signaling pathway (hsa04910)", "Sphingolipid signaling pathway (hsa04071)", "Human immunodeficiency virus 1 infection (hsa05170)" and "Human T-cell leukemia virus 1 infection (hsa05166)" (Figure 4).

\section{Identification of the Chemical Constituents in Yin-Huo-Tang}

Figure 5 given a typical total ion chromatograms (TICs) of non-volatile components extracted by YHT. Although TICs are complex, most chromatographic peaks can be well separated. Chemical compositions in YHT water extracts determined were shown in Additional file 7-9: Table S5-7. Three of the four pivotal active ingredients obtained from the network pharmacology analysis were identified through qualitative phytochemical analysis, namely stigmasterol, nootkatone and ergotamine.

\section{In vivo Validation of the Efficacy of Yin-Huo-Tang}

We established a lewis lung cancer mouse model and evaluated the potential therapeutic effect of YHT.

During the first 7 days of treatment, the weights of all animals gradually increased. During the last 7 days of treatment, the weights of the model and Cisplatin-treated mouses decreased. During the last 3 days of treatment, the body weights of YHT-L, YHT-M and YHT-H mouses decreased. On the tenth day, body weight of the Cis group was the lowest $(P<0.05$, Figure 6). However, after 14 days of treatment, there was no significant difference, which was related to the increasing tumor burden. During the drug treatment, the tumor volumes of all mouses gradually increased. On the fourteenth day, tumor volumes of Cisplatintreated and YHT-H-treated mouses were significantly lower than those of the model, YHT-M-treated and YHT-L-treated mouses $(P<0.05$, Figure 6$)$. The number of tumor recurrenced mouses were potentially reduced in the cisplatin and $\mathrm{YHT}-\mathrm{H}$ treatment groups compared to the model group after treatment for 4 days (Table 3). After treatment for 7 days, the tumor had recurred in all mice, which was related to the high invasiveness of lewis lung carcinoma cells. As expected, cisplatin and YHT-H decreased Ki-67 (a marker of cell proliferation) in tumor tissues (Figure 7). We believed that high doses of YHT could significantly inhibit the recurrence of lung adenocarcinoma. 
Table 3

Tumor recurrence information

\begin{tabular}{|c|c|c|c|c|}
\hline Group & Recurrence rate on day 4 & P value * & Recurrence rate on day 7 & P value* \\
\hline Mod & $100 \%$ & - & $100 \%$ & - \\
\hline Cis & $71.4 \%$ & 0.008 & $100 \%$ & - \\
\hline YHT-L & $100 \%$ & - & $100 \%$ & - \\
\hline YHT-M & $100 \%$ & - & $100 \%$ & - \\
\hline YHT-H & $75 \%$ & 0.003 & $100 \%$ & - \\
\hline \multicolumn{5}{|c|}{ "*n versus Mod group } \\
\hline \multicolumn{5}{|c|}{$\begin{array}{l}\text { RNA sequencing was used to explore the mRNA expression differences between tumor tissues in the } \\
\text { model mouses and YHT-H-treated mouses. There were } 2099 \text { differentially expressed genes, including } \\
1041 \text { upregulated and } 1058 \text { downregulated (Figure } 8 \text { ). KEGG analysis was used to analyze the } \\
\text { functional enrichment of important modules. The top } 15 \text { potential signaling pathways were listed in } \\
\text { Figure 8. "Sphingolipid signaling pathway" and "Non-alcoholic fatty liver disease (NAFLD) pathway" } \\
\text { were predicted by RNA sequencing and network pharmacological analysis at the same time. } \\
\text { Sphingolipid signaling pathway was related to cell survival proliferation, migration and cytoskeletal } \\
\text { events (Figure 9). Then we verified the inhibition of YHT-H for Sphingolipid signaling pathway by PCR } \\
\text { and WB analysis. As expected, YHT-H suppressed lewis lung cancer cells proliferation by inhibiting } \\
\text { S1P/S1PR5/Gi/Ras/Raf/MEK/ERK pathway, and inhibited migration through } \\
\text { S1P/S1PR5/Gi/PI3K/RAC pathway (Figure 10). YHT could inhibit the recurrence of LUAD by } \\
\text { modulating the Sphingolipid signaling pathway. }\end{array}$} \\
\hline
\end{tabular}

\section{Molecular Docking}

Sphingolipid signaling pathway was the common main pathways in network pharmacology and RNA sequencing results. S1PR5 was a key gene in Sphingolipid signaling pathway, and was a hub gene selected by network pharmacology analysis. We found 3-Phenyldecane (Compound CID: 20740), alphaCuparenol (Compound CID: 5316200), cuparene (Compound CID: 86895), 4-Phenylbicyclo [2.2.2] octan-1Ol (Compound CID: 327096) were related with S1PR5 according to network pharmacology analysis (Supplementary Table 4). Stigmasterol (MOL ID: MOL000449), epiguaipyridine (Compound CID: 5317082), nootkatone (Compound CID: 1268142), ergotamine (Compound CID: 8223) were the four pivotal active ingredients. The above 8 substances were docking with S1PR5 respectively. All of them could bind well to S1PR5, as showed in Figure 11, and the detail was listed in Table 4. 
Table 4

Docking results.

\begin{tabular}{|lll|}
\hline Gene name & Active ingredients & Affinity $(\mathrm{kcal} / \mathrm{mol})$ \\
\hline S1PR5(7EW1) & Stigmasterol & -7.2 \\
\cline { 2 - 3 } & Epiguaipyridine & -6.5 \\
\cline { 2 - 3 } & Nootkatone & -6.3 \\
\cline { 2 - 3 } & Ergotamine & -10.2 \\
\hline 3-Phenyldecane & -4.8 \\
\hline Alpha-Cuparenol & -6.8 \\
\hline & Cuparene & -6.6 \\
\cline { 2 - 2 } & 4-Phenylbicyclo & -6.6 \\
\hline
\end{tabular}

\section{Discussion}

Tumor recurrence or metastasis is responsible for lung cancer death. It has been reported that TCM is potentially effective in the prevention of cancer relapse [30-32]. YHT is a classic traditional Chinese prescription used for prevention LUAD recurrence by "nourishing yin and clearing heat". In this study, we predicted the possible pharmacological mechanism of YHT through a network pharmacology approach, and carried out experiment applying lewis lung carcinoma mouses to identify it.

Through a web-based pharmacology approach, 128 potential active ingredients and 419 genetic targets related to LUAD recurrence were identified. S1PR5, PTGER3, OPRM1, OPRK1, MTNR1B, MTNR1A, HTR1D, HTR1B, HTR1A, DRD4, DRD3, DRD2, CXCR4, CX3CR1, CNR2, CNR1, CHRM2, ANXA1, ADRA2C, ADRA2B, ADRA2A, ADORA3, ADORA1, ADCY1, ADRB2, F2, PIK3CA, PIK3R1 were considered to be hub genes (Figure 2). Stigmasterol, epiguaipyridine, nootkatone and ergotamine were identified as pivotal active ingredients. We also revealed several meaningful signaling pathways including "Purine metabolism", "AMPK signaling pathway", "Human cytomegalovirus infection", "Apoptosis", "Insulin signaling pathway", "Sphingolipid signaling pathway" and "Human T-cell leukemia virus 1 infection" (Figure 4). These pathways are mainly related to inflammation, metabolism, immune responses, and apoptosis.

Qualitative phytochemical analysis of the water extract of YHT confirmed the presence of anthraquinone, polyol, phytosterol, terpene, aldehyde, carboxylic compounds, sugar acid, and vitamins (Figure 5). Three of the four pivotal active ingredients also existed in the water extract of YHT, namely stigmasterol, nootkatone and ergotamine. It has been reported that stigmasterol could cause several cancer cell apoptosis, such as ovarian cancer [33], gastric cancer[34] and cholangiocarcinoma [35]. However, the role of stigmasterol in lung cancer was rarely reported. Nootkatone inhibited growth of A549 cells and induced G1 cell cessation by activating AMPK via LKB1-independent and CAMKK2-dependent pathways [36]. Ergotamine was a vasoconstricting agent, which could cause toxicity and inhibit tumor growth [37]. 
The anti-tumor mechanism of ergotamine was unclear yet, and it was worthy of further investigation. There were some other active ingredients identified in the water extract of YHT had direct anticancer effects or inhibited recrudescence and metastasis. P-cymene inhibited A549 cell proliferation through DNA damaging [38]. Beta sitosterol perturbed disturbed cell cycle and induced apoptosis of A549 cells [39], and inhibited H1299 proliferation [40]. Ophiopogonin B inhibited metastasis of A549 cells via the linc00668/miR-432-5p/EMT axis [41]. Ophiopogonin D blocked proliferation of human lung-cancer cells through the suppression of NF-KB, PI3K/AKT, and AP-1 pathways [42]. Pachymic acid induced apoptosis via activating ROS-dependent JNK and ER stress pathways in lung cancer cells [43]. Linalool inhibited A549 cell migration and induced cell growth inhibition and mitochondrial depolarization [44].

In vivo experiments confirmed $\mathrm{YHT}-\mathrm{H}$ reduced the postoperative recurrence of lewis lung carcinoma and inhibited tumor cell proliferation (Figure 6,7). This dose was the dose documented in "Bian Zheng Qi Wen". RNA sequencing was performed to elaborate the mechanism of YHT acting on lewis lung adenocarcinoma recurrence. RNA sequencing, mRNA and protein expression of tumor tissues confirmed sphingolipid signaling pathway was one of the most important mechanisms of YHT treating LUAD recurrence (Figure 8,9). S1P played an important role in normal physiology, inflammation and carcinogenesis [45]. S1P could act on S1P receptors to promote tumorigenesis, and operate between cancer cells and fibroblasts to prompt metastasis of cancer cells [46]. S1P receptors unusual expressed in various cancers, such as colon cancer [47], bladder cancer [48], oral cancer [49]. And it had been reported that S1PR5 had the highest expression level among all the S1P receptors in malignant human tissues [50]. The mitogen-activated protein kinase (MAPK) pathway was a key cell signaling pathway involved in regulating cellular growth, proliferation, and survival. The RAC1 was critical for cellular adhesion, migration, motility and cell proliferation [51]. PI3K/AKT signaling pathway induced RAC1 expression and activity, and then enhanced cell proliferation, survival, migration and metastasis [52]. MAPK pathway [53-55] and RAC1 [56,57] were the focuses of the development of new anti-tumor drugs. YHT-H suppressed lewis lung cancer cells proliferation by inhibiting S1P/S1PR5/Gi/Ras/Raf/MEK/ERK pathway, and inhibited migration through S1P/S1PR5/Gi/PI3K/RAC pathway (Figure 10).

Molecular Docking showed S1PR5 could docking well with the four pivotal active ingredients and other four active ingredients obtained from TCMSP and BATMAN-TCM databases. These further proved that YHT suppressed lung cancer recurrence by inhibiting the sphingolipid signaling pathway. Combined with the results of qualitative phytochemical analysis, we believed stigmasterol, nootkatone and ergotamine played significant roles.

\section{Conclusions}

In conclusion, the present study demonstrated the anticancer effect of YHT. To explore the biological mechanism of YHT inhibiting the recurrence of lung adenocarcinoma we did network pharmacology analysis. We concluded that Stigmasterol, nootkatone and ergotamine were the most important pivotal active ingredients. We found 28 hub genes including S1PR5. The mechanism of YHT could be summed up to inflammation, metabolism, immune responses, and apoptosis. Notably, in the enrichment analysis 
of main target genes according to the network pharmacological analysis and differentially regulated makers acquired from Lewis lung carcinoma mouses, sphingolipid signaling pathway was found to be one of the shared pathways. Then we verified YHT suppressing tumor cells proliferation and inhibiting migration through sphingolipid signaling pathway. And Stigmasterol, nootkatone and ergotamine could dock well with S1PR5. These findings provide a new option for the treatment of LUAD recurrence and also provide information for further revealing the mechanisms of YHT (Figure 1).

However, our study was based on network pharmacology research and limited animal experimental research. Cell model and clinical samples were not use to further verify its authenticity. The pivotal active ingredients were verified by qualitative phytochemical analysis and molecular docking, lacked of quantitative analysis and experimental verification. Our discovery should be further validated in future study.

\section{Abbreviations}

LUAD: Lung Adenocarcinoma; YHT: Yin-Huo-Tang; TCM: Traditional Chinese Medicine; EMT: Epithelial to Mesenchymal Transition; PPI: Protein-Protein Interaction; GO: Gene Ontology; KEGG: Kyoto Encyclopedia of Genes and Genomes; DEGs: The Differential Expression Genes; TCMSP: The Traditional Chinese Medicine Systems Pharmacology database and Analysis Platform; BATMAN-TCM: Bioinformatics Analysis Tool for Molecular Mechanism of Traditional Chinese Medicine; DAVID: The Database for Annotation, Visualization, and Integrated Discovery; STRING: The Search Tool for the Retrieval of Interacting Genes; TICs: Typical total ion chromatograms.

\section{Declarations}

\section{Ethics approval and consent to participate}

Not applicable.

\section{Consent for publication}

Not applicable.

\section{Availability of data and materials}

The datasets of RNA sequencing analyzed during the current study are available in the sequence read archive (SRA) repository, named PRJNA761925 (https://www.ncbi.nlm.nih.gov/sra/PRJNA761925).

The other original contributions presented in the study are included in the article/Supplementary Information, further inquiries can be directed to the corresponding authors.

\section{Competing interests}


The authors declare that they have no conflicts of interest.

\section{Funding}

This work was supported by the Fundamental Research Funds for the Central Universities (No. 2020-JYBZDGG-127), the National Key R\&D Program of China (No. 2018YFC1705102) and Capital's Funds for Health Improvement and Research (No. CFH 2018-1-4201).

\section{Authors' contributions}

Dianna Liu, Kaiwen Hu and Quanwang Li designed the experiments. Dianna Liu, Shicheng Lin and Yuan Li participated in the specific experimental process. Dianna Liu and Tian Zhou performed the research and analyzed the data. Dianna Liu wrote the paper. Dianna Liu, Kaiwen Hu, and Quanwang Li drafted the manuscript. All authors approved and agreed to be responsible for all aspects of the work.

\section{Acknowledgements}

Not applicable.

\section{References}

1. Osarogiagbon RU, Veronesi G, Fang W, Ekman S, Suda K, Aerts JG, et al. Early-Stage NSCLC: Advances in Thoracic Oncology 2018. Journal of thoracic oncology : official publication of the International Association for the Study of Lung Cancer. 2019;14(6):968-78.

2. Siegel RL, Miller KD, Jemal A. Cancer statistics, 2019. CA: a cancer journal for clinicians. 2019;69(1):7-34.

3. Takanashi Y, Funai K, Sato S, Kawase A, Tao H, Takahashi Y, et al. Sphingomyelin(d35:1) as a novel predictor for lung adenocarcinoma recurrence after a radical surgery: a case-control study. BMC cancer. 2020;20(1):800.

4. Liu J, Yang X, Lu X, Zhang L, Luo W, Cheng Y, et al. Impact of T-cell receptor and B-cell receptor repertoire on the recurrence of early stage lung adenocarcinoma. Experimental cell research. 2020;394(2):112134.

5. Yao J, Xue X, Qu D, Westphalen CB, Ge Y, Zhang L, et al. Reverse engineering a predictive signature characterized by proliferation, DNA damage, and immune escape from stage I lung adenocarcinoma recurrence. Acta biochimica et biophysica Sinica. 2020;52(6):638-53.

6. Park IK, Hyun K, Kim ER, Park S, Kang CH, Kim YT. The prognostic effect of the epidermal growth factor receptor gene mutation on recurrence dynamics of lung adenocarcinoma. European journal of cardio-thoracic surgery : official journal of the European Association for Cardio-thoracic Surgery. 2018;54(6):1022-7. 
7. Schwalk AJ, Ost DE, Saltijeral SN, De La Garza H, Casal RF, Jimenez CA, et al. Risk Factors for and Time to Recurrence of Symptomatic Malignant Pleural Effusion in Patients With Metastatic NonSmall Cell Lung Cancer with EGFR or ALK Mutations. Chest. 2021;159(3):1256-64.

8. Patnaik SK, Cortes EG, Kannisto ED, Punnanitinont A, Dhillon SS, Liu S, et al. Lower airway bacterial microbiome may influence recurrence after resection of early-stage non-small cell lung cancer. The Journal of thoracic and cardiovascular surgery. 2021;161(2):419-29.e16.

9. Cho J, Min HY, Pei H, Wei X, Sim JY, Park SH, et al. The ATF6-EGF Pathway Mediates the Awakening of Slow-Cycling Chemoresistant Cells and Tumor Recurrence by Stimulating Tumor Angiogenesis. Cancers. 2020;12(7).

10. Gong FL, Wang L, Yu LG, Dang YF, Jiang XN, Zhao L, et al. DHPAC, a novel microtubule depolymerizing agent, suppresses angiogenesis and vasculogenic mimicry formation of human nonsmall cell lung cancer. Journal of cellular biochemistry. 2020.

11. Ni L, Li Z, Shi X, Yao C, Sun J, Ai M, et al. Rosthorin A inhibits non-small cell lung cancer cell growth and metastasis through repressing epithelial-mesenchymal transition via downregulating Slug. Anti-cancer drugs. 2020;31(10):997-1003.

12. Reno TA, Kim JY, Raz DJ. Triptolide Inhibits Lung Cancer Cell Migration, Invasion, and Metastasis. The Annals of thoracic surgery. 2015;100(5):1817-24; discussion 24-5.

13. Xu Z, Zhang F, Zhu Y, Liu F, Chen X, Wei L, et al. Traditional Chinese medicine Ze-Qi-Tang formula inhibit growth of non-small-cell lung cancer cells through the p53 pathway. Journal of ethnopharmacology. 2019;234:180-8.

14. Liu R, Zheng H, Li W, Guo Q, He S, Hirasaki Y, et al. Anti-tumor enhancement of Fei-Liu-Ping ointment in combination with celecoxib via cyclooxygenase-2-mediated lung metastatic inflammatory microenvironment in Lewis lung carcinoma xenograft mouse model. Journal of translational medicine. 2015;13:366.

15. Yao J, Jiao L, Yao Y, Lu Y, Shi J, Li J, et al. The effect of comprehensive rehabilitation program plus chemotherapy on quality of life in patients with postoperative non-small-cell lung cancer: study protocol of a multicenter randomized clinical trial. Trials. 2020;21(1):309.

16. Wang K, Chen Q, Shao Y, Yin S, Liu C, Liu Y, et al. Anticancer activities of TCM and their active components against tumor metastasis. Biomedicine \& pharmacotherapy = Biomedecine \& pharmacotherapie. 2021;133:111044.

17. Wang XQ, Zhang Y, Hou W, Wang YT, Zheng JB, Li J, et al. Association between Chinese Medicine Therapy and Survival Outcomes in Postoperative Patients with NSCLC: A Multicenter, Prospective, Cohort Study. Chinese journal of integrative medicine. 2019;25(11):812-9. 
18. Zhao X, Dai X, Wang S, Yang T, Yan Y, Zhu G, et al. Traditional Chinese Medicine Integrated with Chemotherapy for Stage II-IIIA Patients with Non-Small-Cell Lung Cancer after Radical Surgery: A Retrospective Clinical Analysis with Small Sample Size. Evidence-based complementary and alternative medicine : eCAM. 2018;2018:4369027.

19. Cheng Y, Duan S, Zuo M, Hu K. Experience in Treating Tumor with Yinhuo Decoction Under the Mode of Green Therapy of Cancer. China Medical Herald. 2018;37(07):839-42.

20. Wang M, Hu K, Liu C, Zhou T. Treatment thought of malignant tumor based on the kidney tonification for anti-aging. China Journal of Traditional Chinese Medicine and Pharmacy. 2019;34(06):2333-6.

21. Wang M, Zhou T, Hu K. Application of Yinhuo Decoction in Coping with Side Effects of $\triangle$ adiotherapy and Chemotherapy of Malignant Tumor. Acta Chinese Medicine. 2019;34(06):1156-8+87.

22. Li L, Dai W, Li W, Zhang Y, Wu Y, Guan C, et al. Integrated Network Pharmacology and Metabonomics to Reveal the Myocardial Protection Effect of Huang-Lian-Jie-Du-Tang on Myocardial Ischemia. Frontiers in pharmacology. 2020;11:589175.

23. Zhang Z, Yi P, Yang J, Huang J, Xu P, Hu M, et al. Integrated network pharmacology analysis and serum metabolomics to reveal the cognitive improvement effect of Bushen Tiansui formula on Alzheimer's disease. Journal of ethnopharmacology. 2020;249:112371.

24. Chen RB, Yang YD, Sun K, Liu S, Guo W, Zhang JX, et al. Potential mechanism of Ziyin Tongluo Formula in the treatment of postmenopausal osteoporosis: based on network pharmacology and ovariectomized rat model. Chinese medicine. 2021;16(1):88.

25. UniProt C. UniProt: a hub for protein information. Nucleic acids research. 2015;43(Database issue):D204-12.

26. Yang WJ, Wang HB, Wang WD, Bai PY, Lu HX, Sun CH, et al. A network-based predictive gene expression signature for recurrence risks in stage II colorectal cancer. Cancer medicine. 2020;9(1):179-93.

27. Li X, Tang H, Tang Q, Chen W. Decoding the Mechanism of Huanglian Jiedu Decoction in Treating Pneumonia Based on Network Pharmacology and Molecular Docking. Frontiers in cell and developmental biology. 2021;9:638366.

28. Lei G, Zhang Y, Koppula P, Liu X, Zhang J, Lin SH, et al. The role of ferroptosis in ionizing radiation-induced cell death and tumor suppression. Cell research. 2020;30(2):146-62.

29. Liu D, Chen F, Yu X, Xiu L, Liu H, Chen S, et al. Do Different Species of Sargassum in Haizao Yuhu Decoction Cause Different Effects in a Rat Goiter Model? Evidence-based complementary and alternative medicine : eCAM. 2019;2019:5645620. 
30. Winkler C. [Traditional Chinese medicine and cancer recurrence]. Revue de l'infirmiere. 2015(207):43.

31. Wang Y, Li JW, Qin YN, Sun CP, Chen JJ, Ruan YY, et al. Clinical observation on the effect of Chinese medicine-"TCM formula" intervention on recurrence and metastasis of triple negative breast cancer. Complementary therapies in medicine. 2020;52:102456.

32. Liu Q, Hodge J, Wang J, Wang Y, Wang L, Singh U, et al. Emodin reduces Breast Cancer Lung Metastasis by suppressing Macrophage-induced Breast Cancer Cell Epithelial-mesenchymal transition and Cancer Stem Cell formation. Theranostics. 2020;10(18):8365-81.

33. Bae H, Song G, Lim W. Stigmasterol Causes Ovarian Cancer Cell Apoptosis by Inducing Endoplasmic Reticulum and Mitochondrial Dysfunction. Pharmaceutics. 2020;12(6).

34. Li K, Yuan D, Yan R, Meng L, Zhang Y, Zhu K. Stigmasterol exhibits potent antitumor effects in human gastric cancer cells mediated via inhibition of cell migration, cell cycle arrest, mitochondrial mediated apoptosis and inhibition of JAK/STAT signalling pathway. Journal of BUON : official journal of the Balkan Union of Oncology. 2018;23(5):1420-5.

35. Kangsamaksin T, Chaithongyot S, Wootthichairangsan C, Hanchaina R, Tangshewinsirikul C, Svasti J. Lupeol and stigmasterol suppress tumor angiogenesis and inhibit cholangiocarcinoma growth in mice via downregulation of tumor necrosis factor-a. PloS one. 2017;12(12):e0189628.

36. Hung LVM, Moon JY, Ryu JY, Cho SK. Nootkatone, an AMPK activator derived from grapefruit, inhibits KRAS downstream pathway and sensitizes non-small-cell lung cancer A549 cells to adriamycin. Phytomedicine : international journal of phytotherapy and phytopharmacology. 2019;63:153000.

37. Mrusek M, Seo EJ, Greten HJ, Simon M, Efferth T. Identification of cellular and molecular factors determining the response of cancer cells to six ergot alkaloids. Investigational new drugs. 2015;33(1):3244.

38. Özkan A, Erdoğan A. Evaluation of cytotoxic, membrane, and DNA damaging effects of Thymus revolutus Célak essential oil on different cancer cells. Turkish journal of medical sciences. 2017;47(2):702-14.

39. Rajavel T, Mohankumar R, Archunan G, Ruckmani K, Devi KP. Beta sitosterol and Daucosterol (phytosterols identified in Grewia tiliaefolia) perturbs cell cycle and induces apoptotic cell death in A549 cells. Scientific reports. 2017;7(1):3418.

40. Anwar MA, Tabassam S, Gulfraz M, Sheeraz Ahmad M, Raja GK, Arshad M. Isolation of Oxyberberine and $\beta$-Sitosterol from Berberis lycium Royle Root Bark Extract and In Vitro Cytotoxicity against Liver and Lung Cancer Cell Lines. Evidence-based complementary and alternative medicine : eCAM. 2020;2020:2596082. 
41. Hu C, Jiang R, Cheng Z, Lu Y, Gu L, Li H, et al. Ophiopogonin-B Suppresses Epithelialmesenchymal Transition in Human Lung Adenocarcinoma Cells via the Linc00668/miR-432-5p/EMT Axis. Journal of Cancer. 2019;10(13):2849-56.

42. Lee JH, Kim C, Lee SG, Yang WM, Um JY, Sethi G, et al. Ophiopogonin D modulates multiple oncogenic signaling pathways, leading to suppression of proliferation and chemosensitization of human lung cancer cells. Phytomedicine : international journal of phytotherapy and phytopharmacology. 2018;40:165-75.

43. Ma J, Liu J, Lu C, Cai D. Pachymic acid induces apoptosis via activating ROS-dependent JNK and ER stress pathways in lung cancer cells. Cancer cell international. 2015;15:78.

44. Rodenak-Kladniew B, Castro MA, Crespo R, Galle M, García de Bravo M. Anti-cancer mechanisms of linalool and 1,8-cineole in non-small cell lung cancer A549 cells. Heliyon. 2020;6(12):e05639.

45. Sattar RSA, Sumi MP, Nimisha, Apurva, Kumar A, Sharma AK, et al. S1P signaling, its interactions and cross-talks with other partners and therapeutic importance in colorectal cancer. Cellular signalling. 2021;86:110080.

46. Pyne NJ, El Buri A, Adams DR, Pyne S. Sphingosine 1-phosphate and cancer. Advances in biological regulation. 2018;68:97-106.

47. Zhou H, Yin X, Bai F, Liu W, Jiang S, Zhao J. The Role and Mechanism of S1PR5 in Colon Cancer. Cancer management and research. 2020;12:4759-75.

48. Palangi A, Shakhssalim N, Parvin M, Bayat S, Allameh A. Differential expression of S1P receptor subtypes in human bladder transitional cell carcinoma. Clinical \& translational oncology : official publication of the Federation of Spanish Oncology Societies and of the National Cancer Institute of Mexico. 2019;21(9):1240-9.

49. da Silva G, de Matos LL, Kowalski LP, Kulcsar M, Leopoldino AM. Profile of sphingolipid-related genes and its association with prognosis highlights sphingolipid metabolism in oral cancer. Cancer biomarkers : section A of Disease markers. 2021;32(1):49-63.

50. Wang C, Mao J, Redfield S, Mo Y, Lage JM, Zhou X. Systemic distribution, subcellular localization and differential expression of sphingosine-1-phosphate receptors in benign and malignant human tissues. Experimental and molecular pathology. 2014;97(2):259-65.

51. Zou T, Mao X, Yin J, Li X, Chen J, Zhu T, et al. Emerging roles of RAC1 in treating lung cancer patients. Clinical genetics. 2017;91(4):520-8.

52. Tan S, Yi P, Wang H, Xia L, Han Y, Wang H, et al. RAC1 Involves in the Radioresistance by Mediating Epithelial-Mesenchymal Transition in Lung Cancer. Frontiers in oncology. 2020;10:649. 
53. Ruscetti M, Leibold J, Bott MJ, Fennell M, Kulick A, Salgado NR, et al. NK cell-mediated cytotoxicity contributes to tumor control by a cytostatic drug combination. Science (New York, NY). 2018;362(6421):1416-22.

54. Subbiah V, Baik C, Kirkwood JM. Clinical Development of BRAF plus MEK Inhibitor Combinations. Trends in cancer. 2020;6(9):797-810.

55. Zhou ZW, Ambrogio C, Bera AK, Li Q, Li XX, Li L, et al. KRAS(Q61H) Preferentially Signals through MAPK in a RAF Dimer-Dependent Manner in Non-Small Cell Lung Cancer. Cancer research. 2020;80(17):3719-31.

56. Murillo MM, Rana S, Spencer-Dene B, Nye E, Stamp G, Downward J. Disruption of the Interaction of RAS with PI 3-Kinase Induces Regression of EGFR-Mutant-Driven Lung Cancer. Cell reports. 2018;25(13):3545-53.e2.

57. Zhang T, Wang N. miR-135a Confers Resistance to Gefitinib in Non-Small Cell Lung Cancer Cells by Upregulation of RAC1. Oncology research. 2018;26(8):1191-200.

\section{Figures}




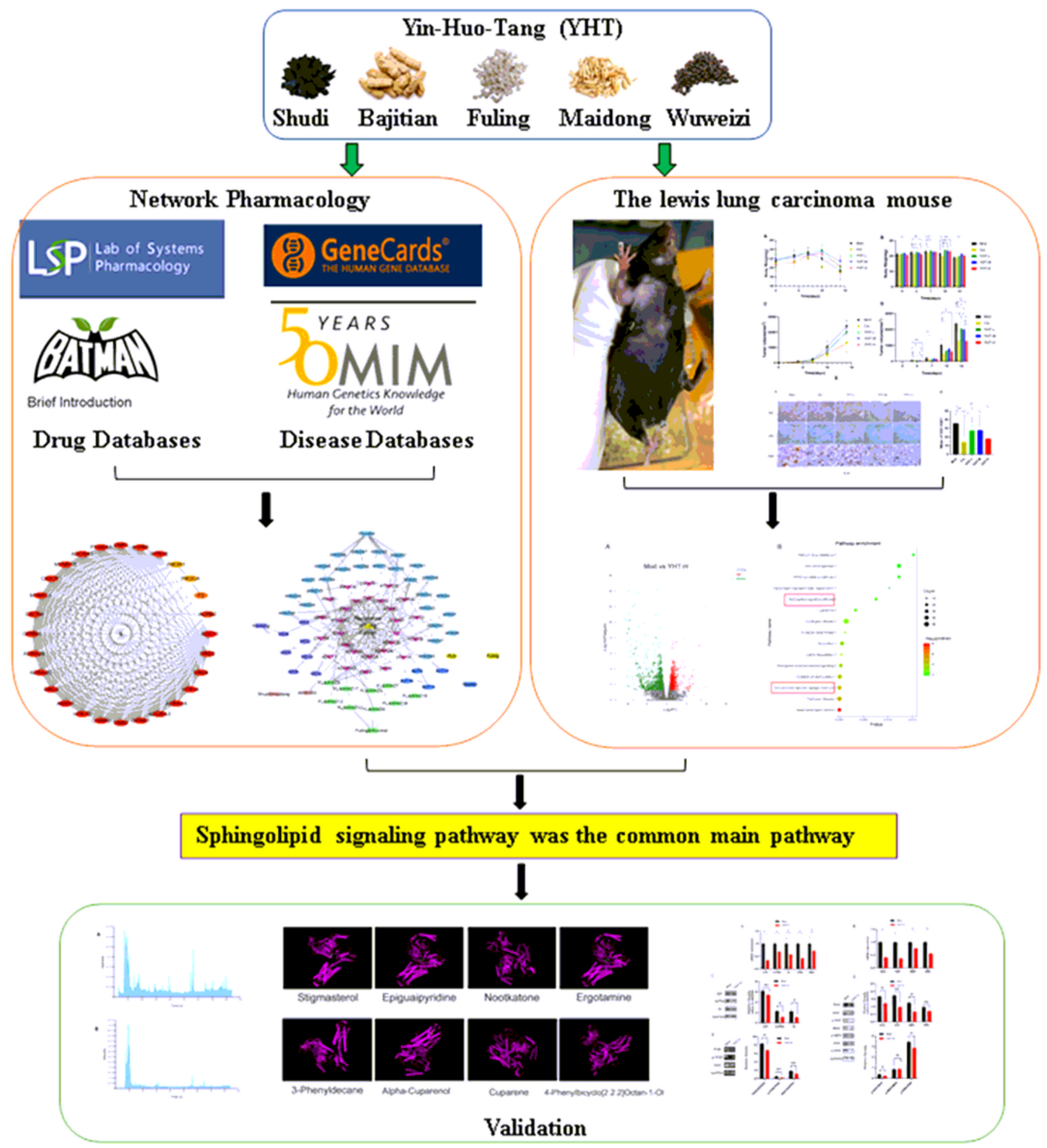

Figure 1

The technical strategy. Firstly, YHT bioactive compounds and related targets were preliminarily understood, and drug-active ingredients-hub gene symbols-disease network was constructed, in order to elucidate their molecular mechanism during LUAD recurrence. Subsequently, the efficacy of YHT was validated in lewis lung carcinoma mouses. Meanwhile, the RNA sequencing was used to explore DEGs thus providing the potential mechanisms. Sphingolipid signaling pathway was predicted by RNA 
sequencing and network pharmacological analysis at the same time. Finally, qualitative phytochemical analysis, molecular docking technology, PCR and WB analysis were used to validate the pivotal active ingredients (Stigmasterol, nootkatone and ergotamine), hub gene (S1PR5) and main pathway (Sphingolipid signaling pathway).
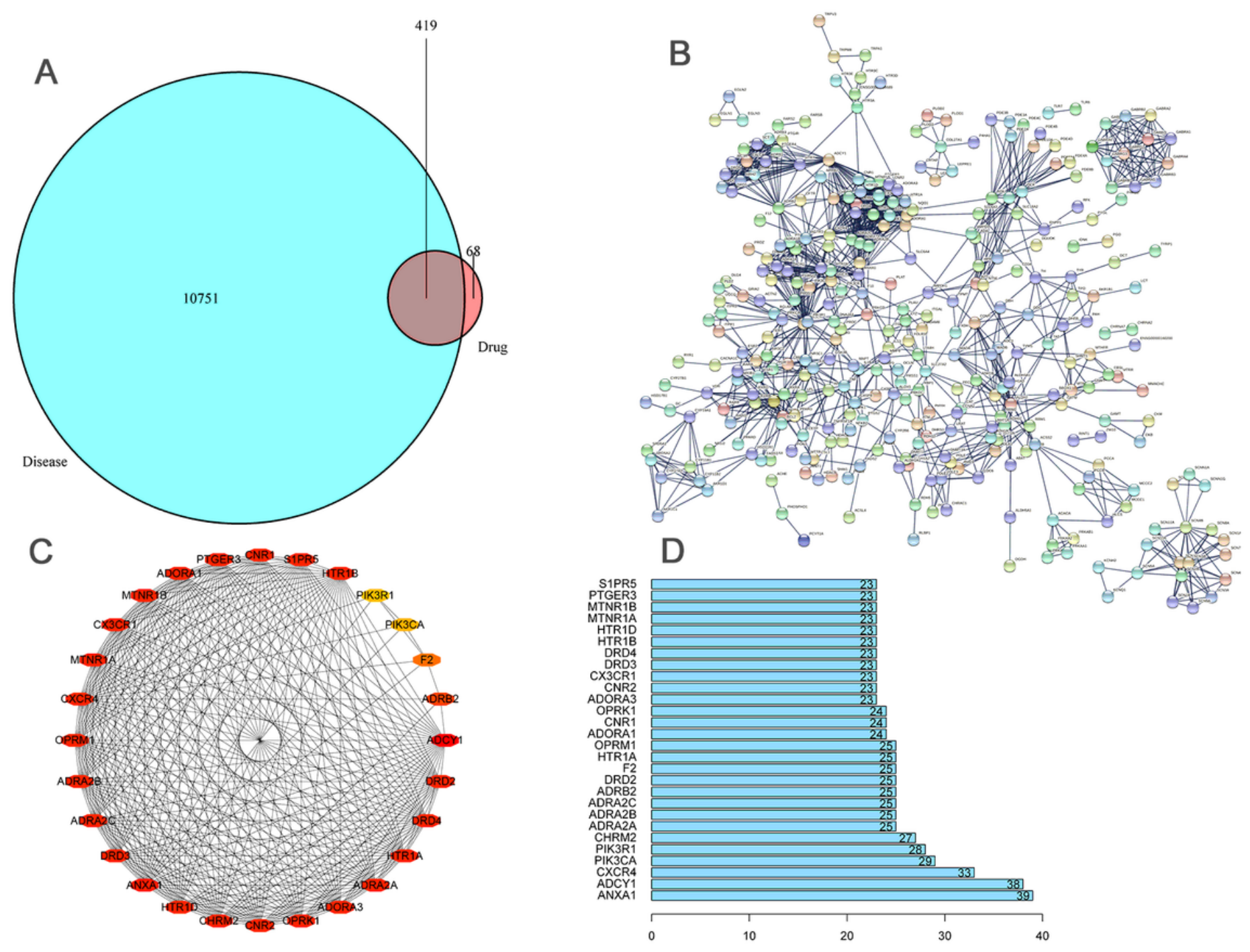

Figure 2

PPI networks. (A) 419 relevant overlapping targets shared between YHT and LUAD recurrence;(B) PPI network of YHT acting on LUAD recurrence (the minimum interaction score was 0.9); (C) and (D) The hub genes evaluated by maximal clique centrality ( $>87178291119$, Figure $2 \mathrm{C}$ ) and the degree ( $>22$, Figure 2D). 


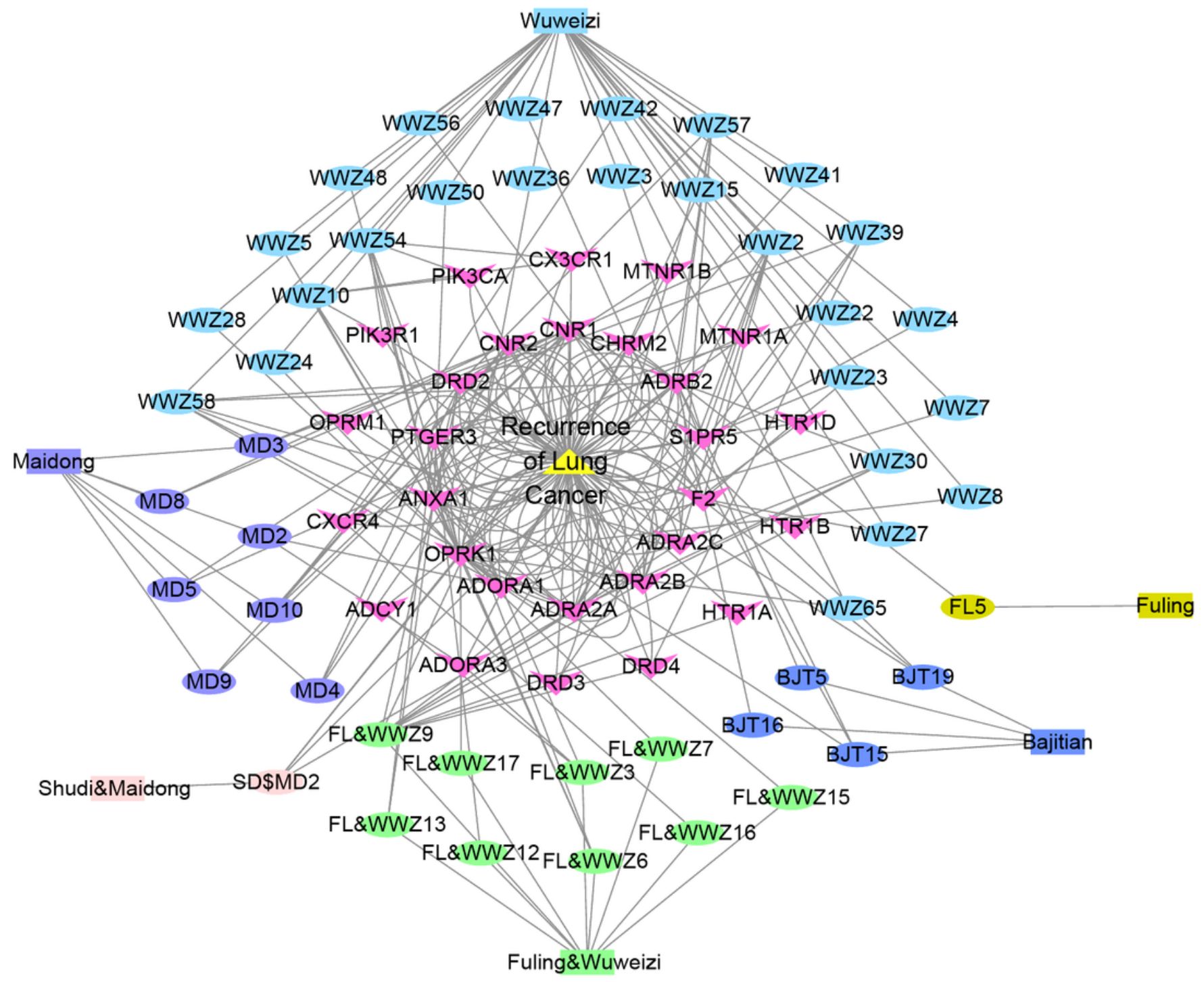

\section{Figure 3}

Drug-Active ingredients-Hub gene symbols-Disease network of YHT in the treatment of LUAD recurrence. The detail of the code in the figure was listed in Additional file 6: Table S4. There were 5 Chinese herbs, 48 active ingredients and 28 hub genes in the network. 
A

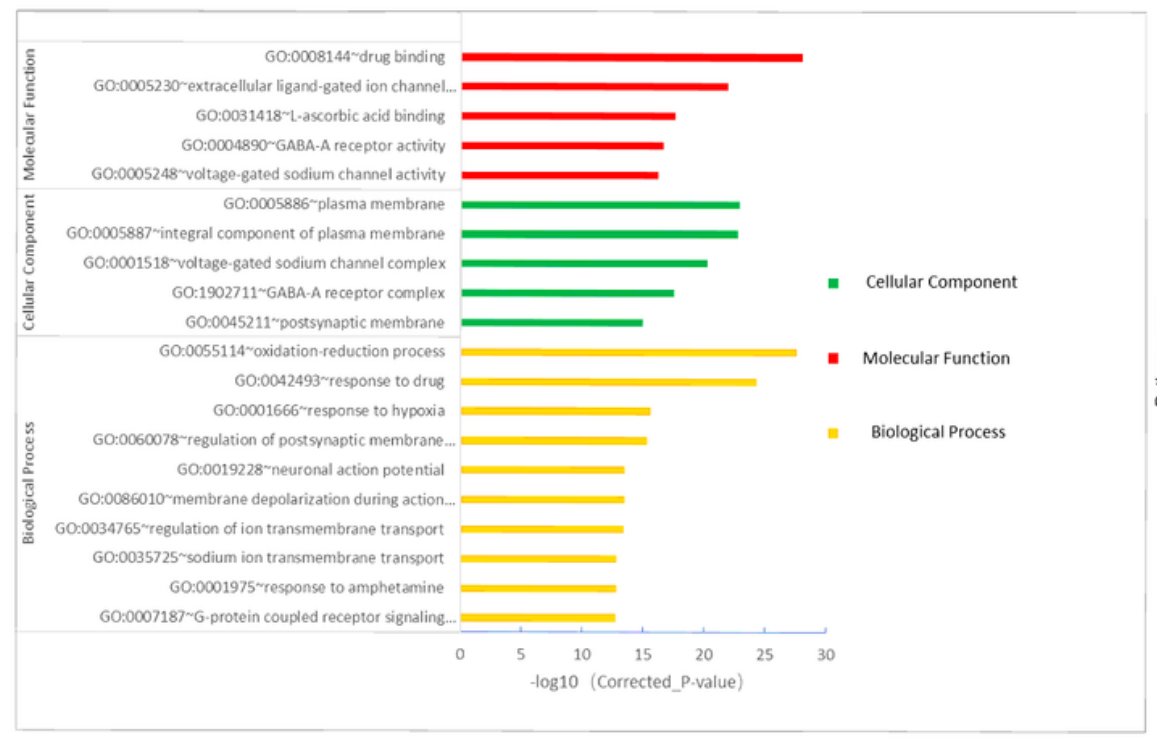

B

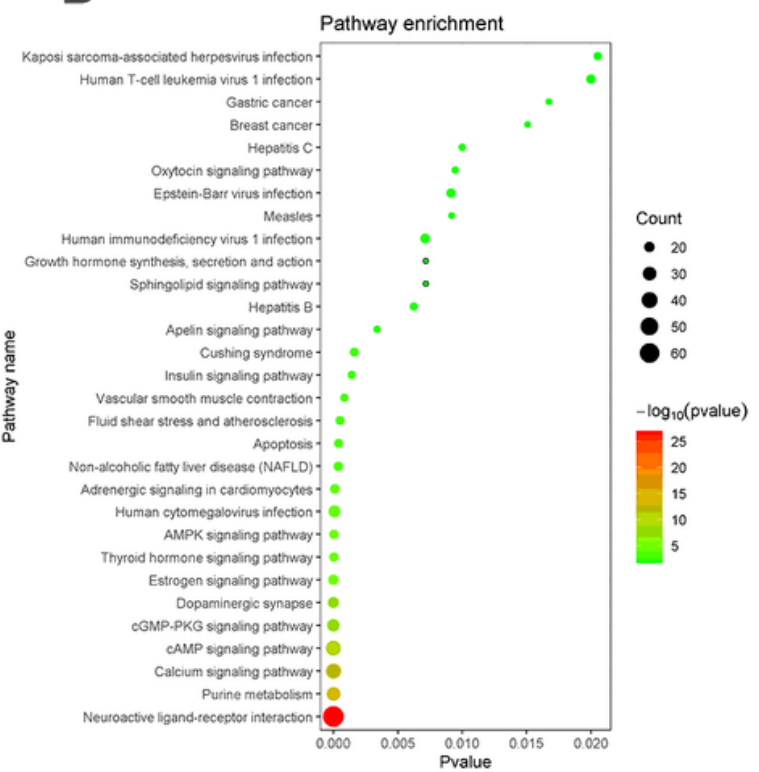

\section{Figure 4}

GO and KEGG enrichment analysis. (A) Barplot showing the BP, CC, and MF domains in the ontology; (B) Dotplot showing the top 30 of KEGG pathways. 

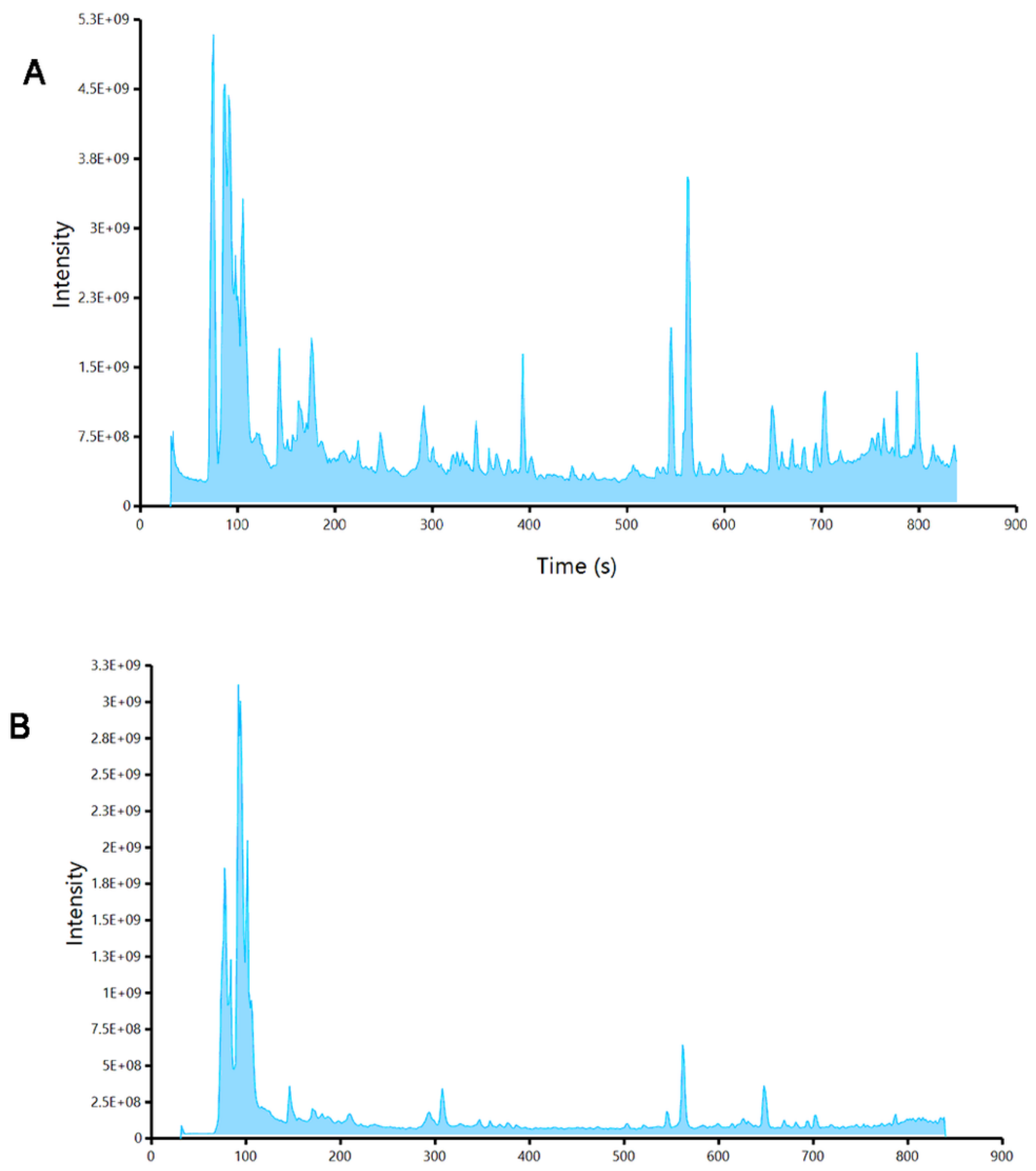

Time (s)

\section{Figure 5}

TICs of the water extract of YHT by UPLC/Q-TOF-MS/MS. (A) TIC of YHT in positive ion mode; (B) TIC of $\mathrm{YHT}$ in negative ion mode. 

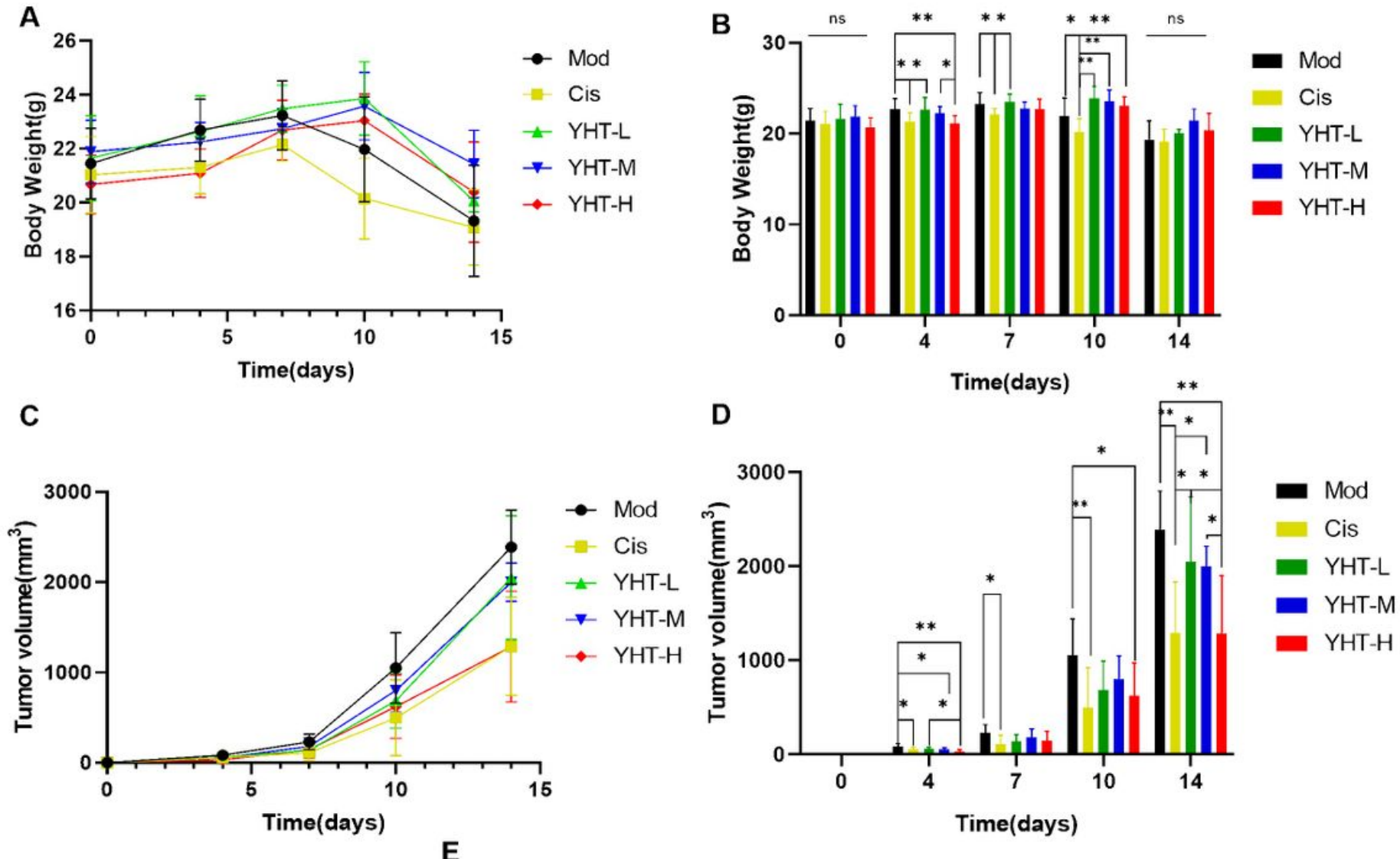

\section{Figure 6}

Body weight, tumor volume of mouses. (A) and (B) On the tenth day, body weight of the Cis group was the lowest. However, there was no significant difference among them after drug treatment for 14 days; (C) and (D)After drug treatment for 14 days, tumor volumes of Cisplatin-treated and YHT-H-treated mouse swere significantly lower than those of the model, YHT-M-treated and YHT-L-treated mouses. Error bars are means $\pm S D, n=10$. $P$ values by one-way analysis of variance. $* P<0.05$ and $* * P<0.01$.

A

Mod

Cis

YHT-L

YHT-M

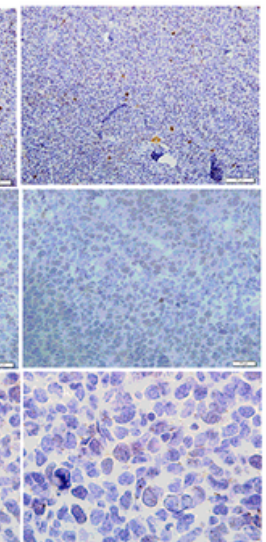

B

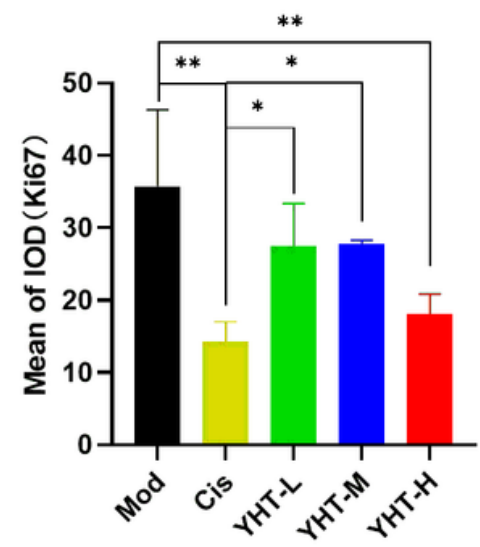




\section{Figure 7}

Immunohistochemical staining (Ki67) of tumor tissues from Lewis lung carcinoma mouses with the indicated treatments. Error bars are means $\pm S D, n=3$ randomly selected magnification fields. $P$ values by one-way analysis of variance. $* \mathrm{P}<0.05$ and $* * \mathrm{P}<0.01$.

A

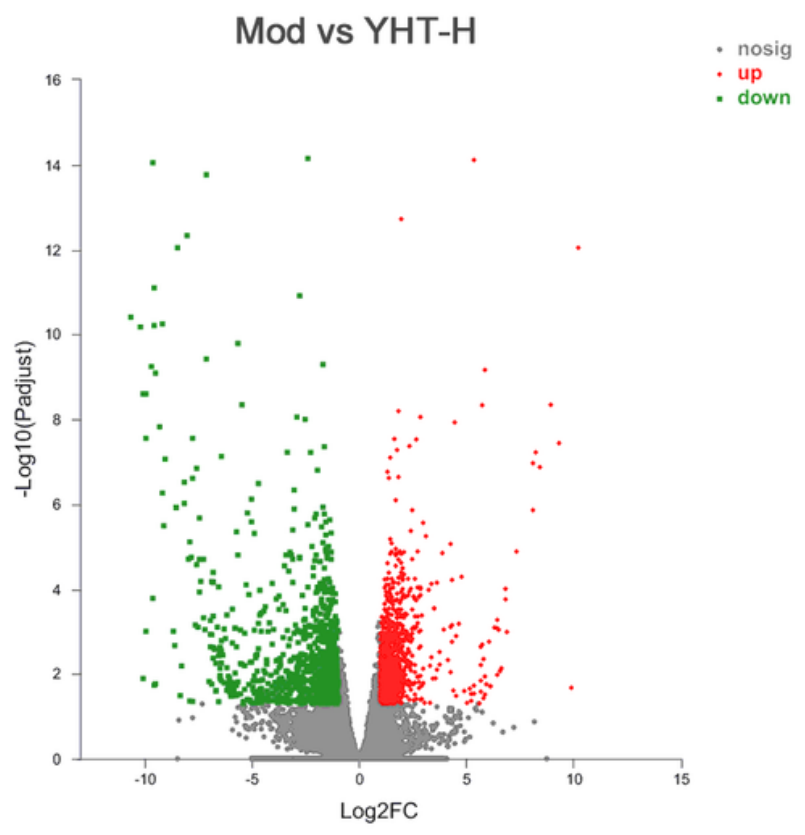

B

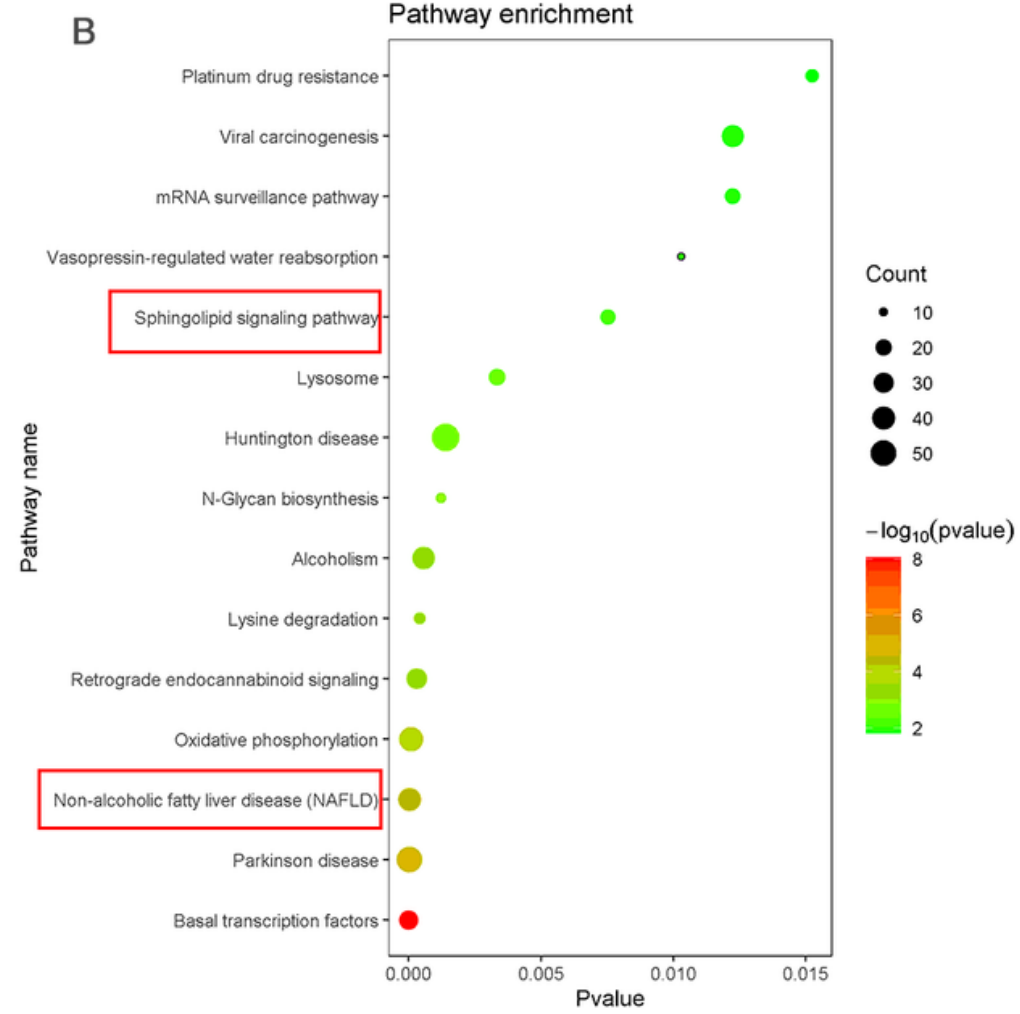

Figure 8

The results of RNA sequencing between tumor tissues in the model mouses and YHT-H-treated mouses. (A)Volcano plot of the DEGs between tumor tissues in the model mouses and YHT-H-treated mouses; (B) Dotplot showing the top 15 of KEGG pathways. "Sphingolipid signaling pathway" and "Non-alcoholic fatty liver disease (NAFLD) pathway" were predicted by RNA sequencing and network pharmacological analysis at the same time. 


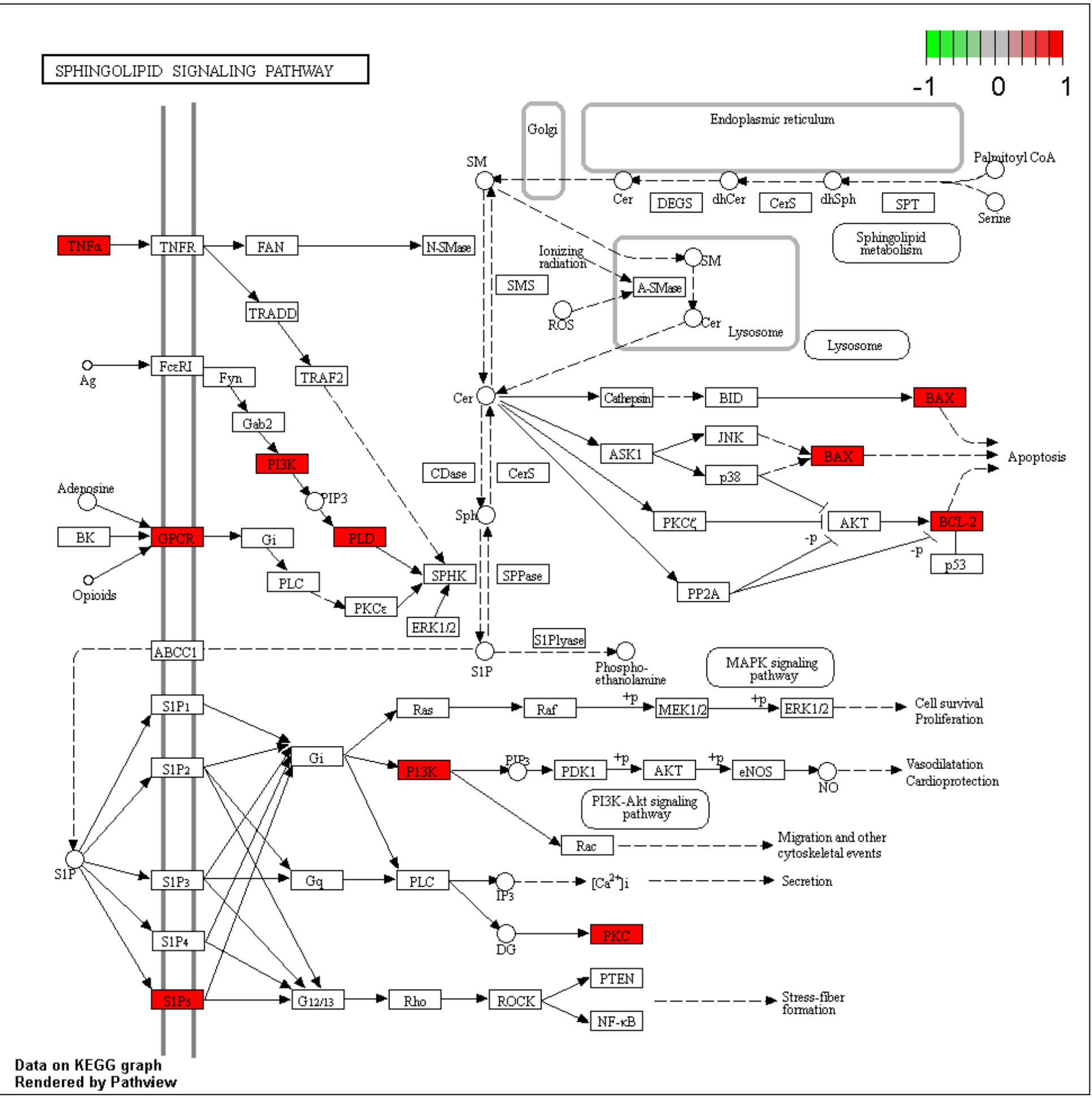

Figure 9

Sphingolipid signaling pathway from KEGG. The genes in red were the relevant overlapping targets shared between YHT and LUAD recurrence. Sphingolipid signaling pathway was related to cell survival proliferation, migration and cytoskeletal events. 
C
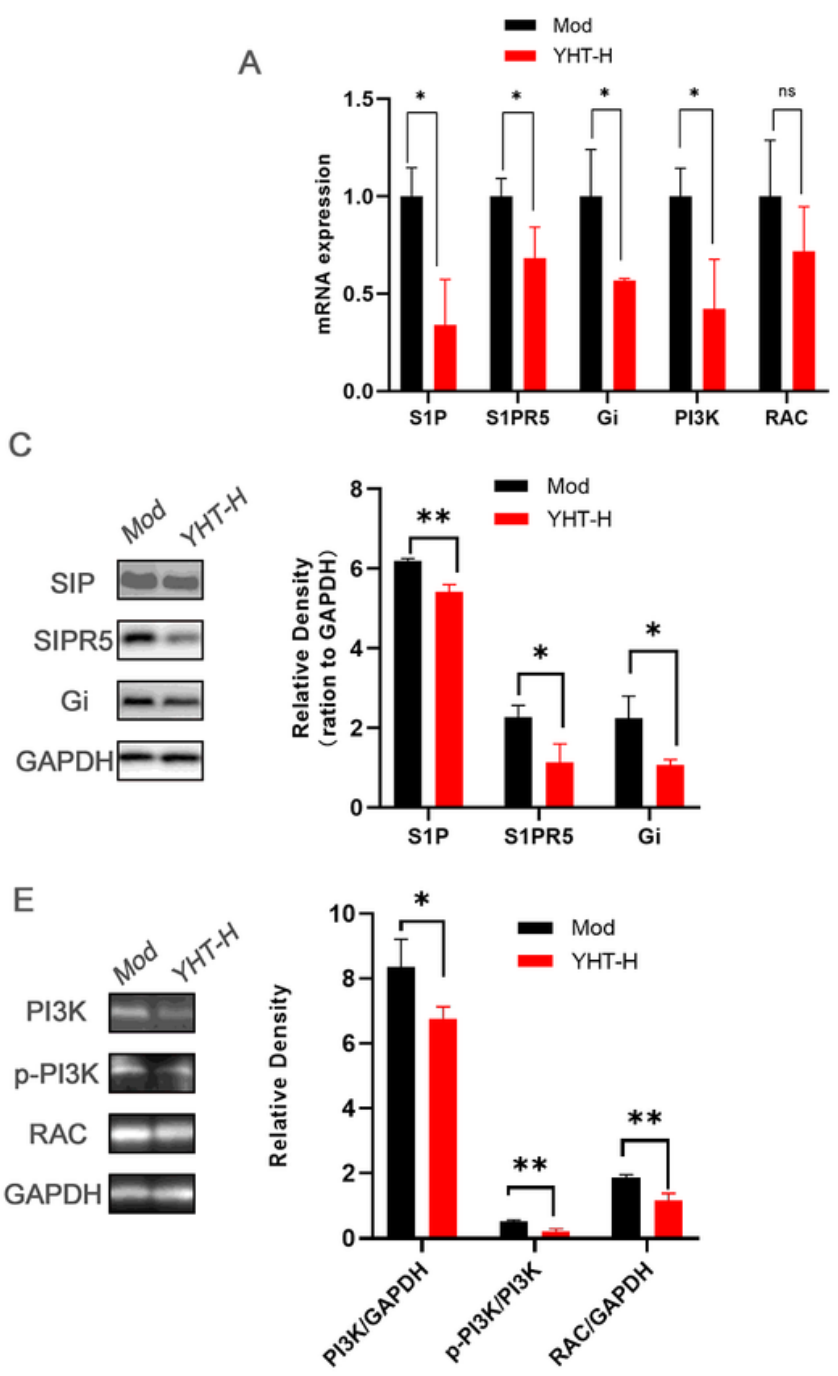

B

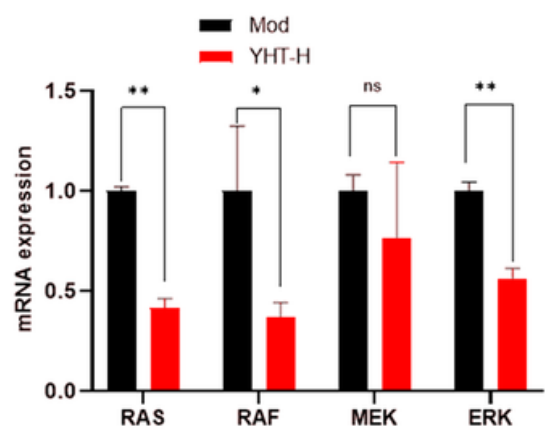

D
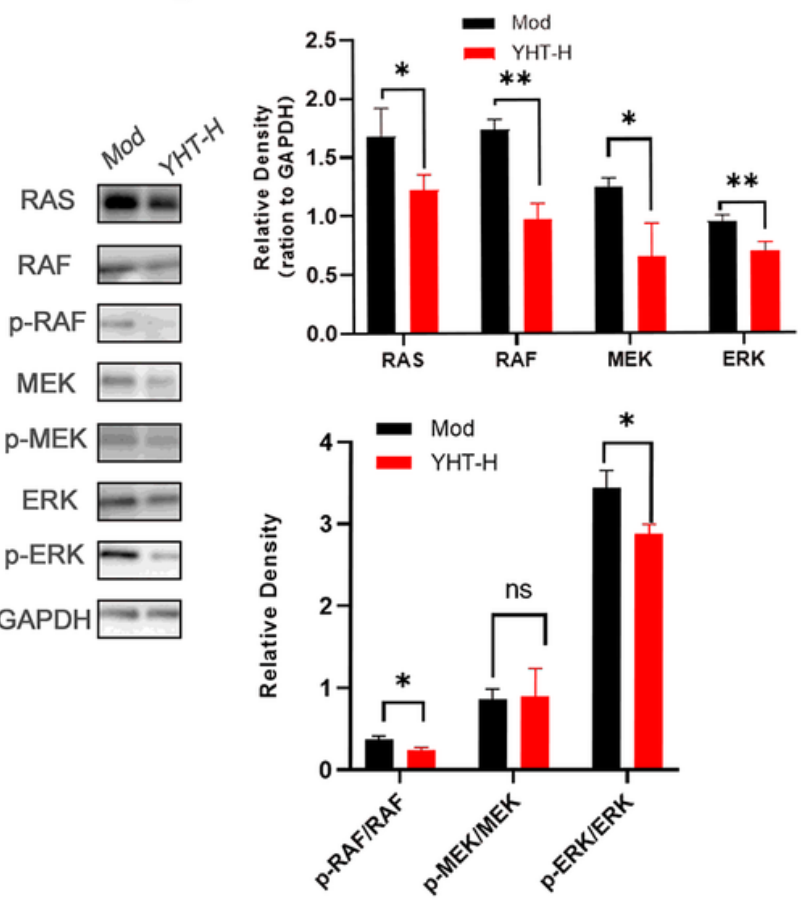

Figure 10

YHT suppressed lewis lung carcinoma recurrence by inhibiting the sphingolipid signaling pathway. (A) and (B) qRT-PCR analysis of S1P, S1PR5, Gi, PI3K, RAC, Ras, Raf, MEK and ERK expression in lewis lung carcinoma mouses after the indicated 14 days treatments;(B) Western blotting analysis of S1P, S1PR5, Gi, PI3K, RAC, Ras, Raf, MEK and ERK expression in lewis lung carcinoma mouses after the indicated 14 days treatments. Error bars are means $\pm S D, n=3$ randomly selected magnification fields. $P$ values were calculated using two-tailed unpaired Student's t-test. $* \mathrm{P}<0.05$ and $* * \mathrm{P}<0.01$. 


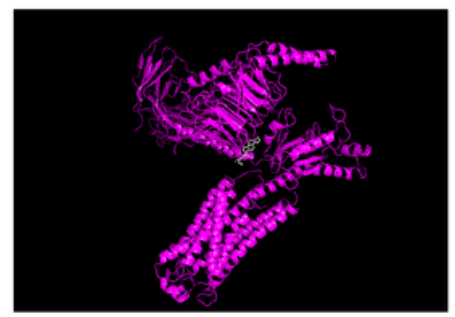

Stigmasterol

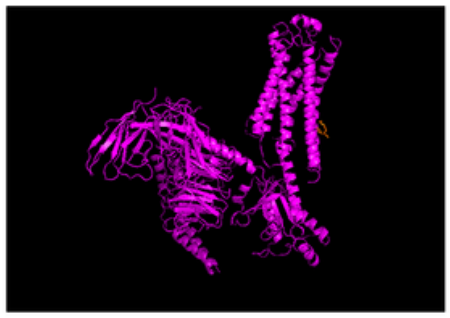

3-Phenyldecane

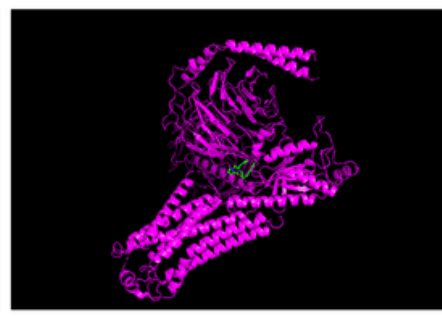

Epiguaipyridine

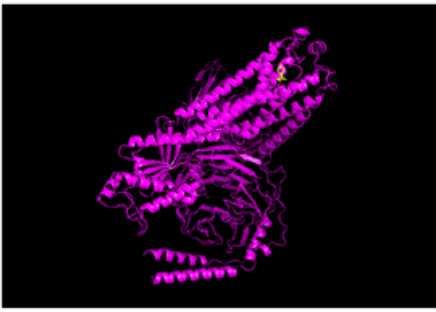

Alpha-Cuparenol

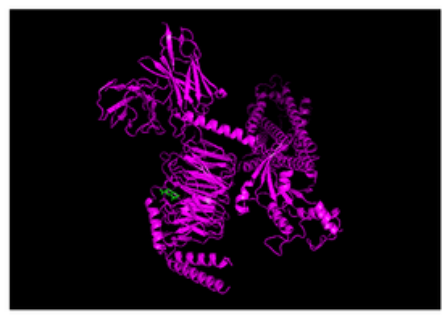

Nootkatone

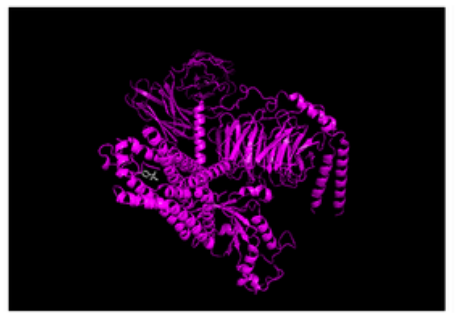

Cuparene
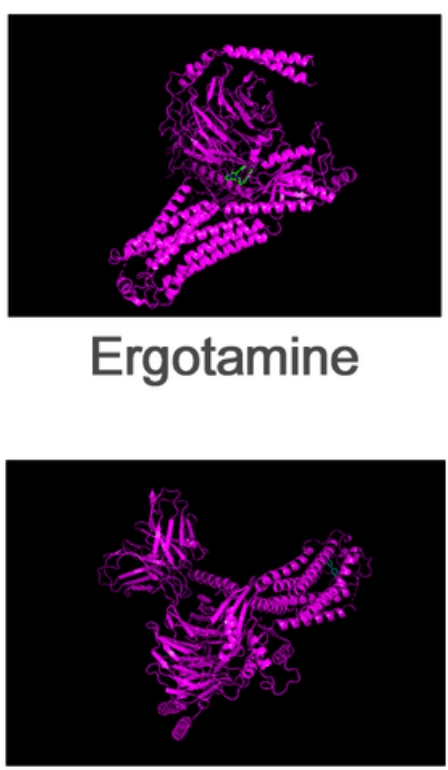

Ergotamine

4-Phenylbicyclo[2.2.2]Octan-1-OI

\section{Figure 11}

Molecular Docking. S1PR5 could docking well with the four pivotal active ingredients and other four active ingredients obtained from TCMSP and BATMAN-TCM databases.

\section{Supplementary Files}

This is a list of supplementary files associated with this preprint. Click to download.

- FigureS1EthicsStatementofAnimalExperimentStudy.pdf

- FigureS2ActiveingredientsTargetNetwork.tif

- TableS1CandidateCcompoundsofYHT.xlsx

- TableS2TargetsofYHTandLUADRecurrence.xIsx

- TableS3DrugActiveingredientsGenesDiseaseNetwork.csv

- TableS4DrugActiveingredientsHubgenesDiseasenetwork.csv

- TableS5LCMSAnalysisofAll.xIsx

- TableS6LCMSAnalysisofMetabolomepos.xlsx

- TableS7LCMSAnalysisofMetabolomeneg.xlsx 